Supporting Information

\title{
Persulfurated Coronene and Its Chalcogenide Analogues: Insight into Effects of the Peripheral Substitution
}

\begin{abstract}
Qing Zhang and Zexing Cao*
State Key Laboratory of Physical Chemistry of Solid Surfaces and Fujian Provincial Key Laboratory of Theoretical and Computational Chemistry, College of Chemistry and Chemical Engineering, Xiamen University, Xiamen 360015, China
\end{abstract}

Corresponding Authors:

*E-mail: zxcao@x,xmu.edu.cn

Table of contents

1. Figures S1 S10

2. Tables S1 S6 


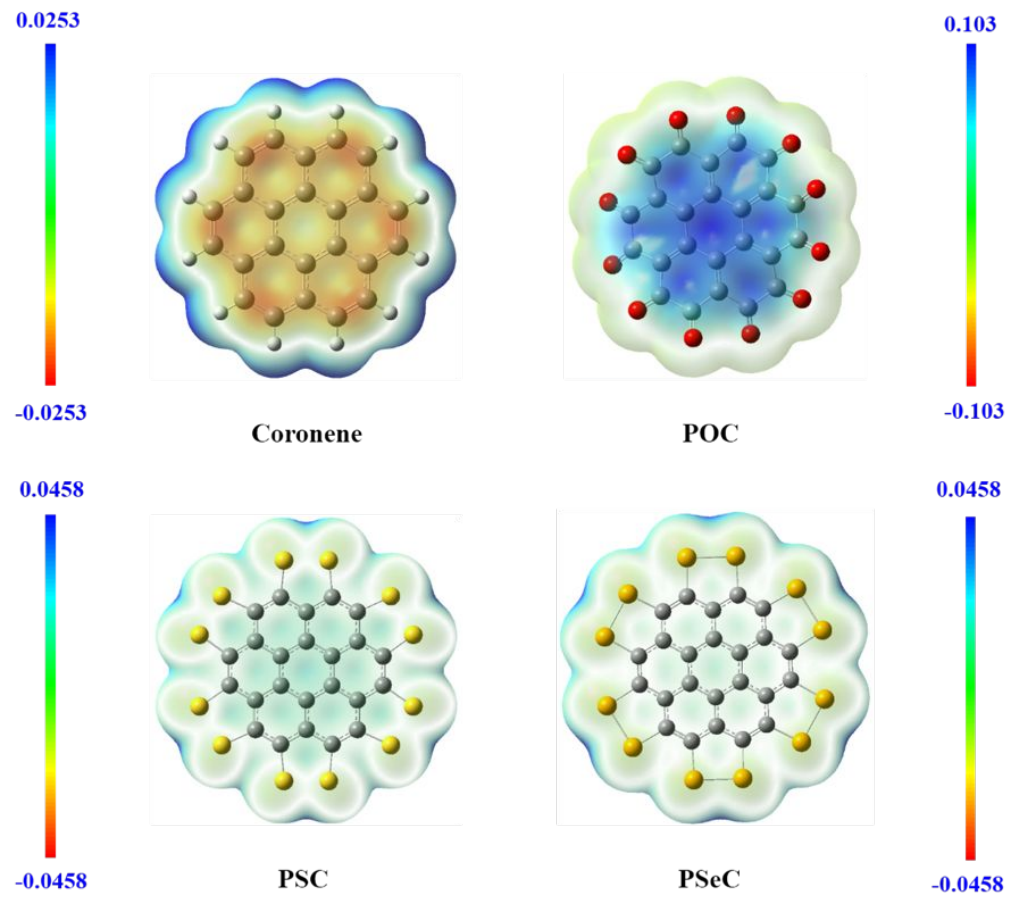

Figure S1. Molecular electrostatic potentials (MEPs) of coronene, POC, PSC and PSeC calculated at the B3LYP/6-31+G(d,p) level. 

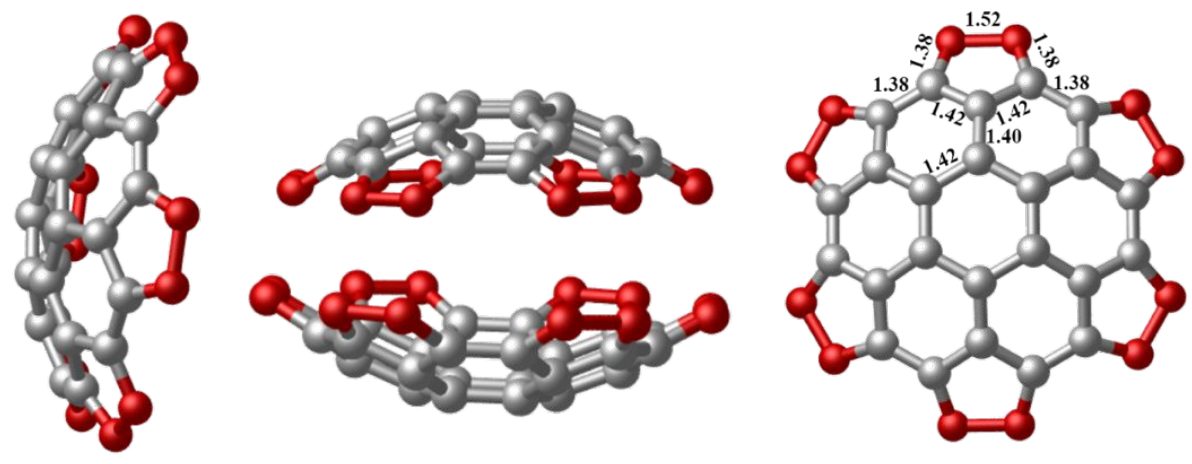

Figure S2. The bowl-shaped structure and key bond lengths $(\AA)$ of the POC molecule. 


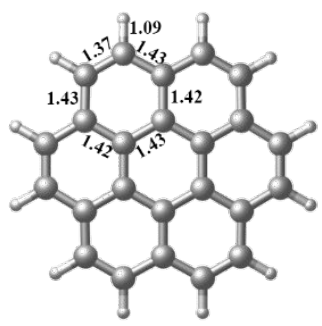

${ }^{1}$ [Coronene $^{0}$
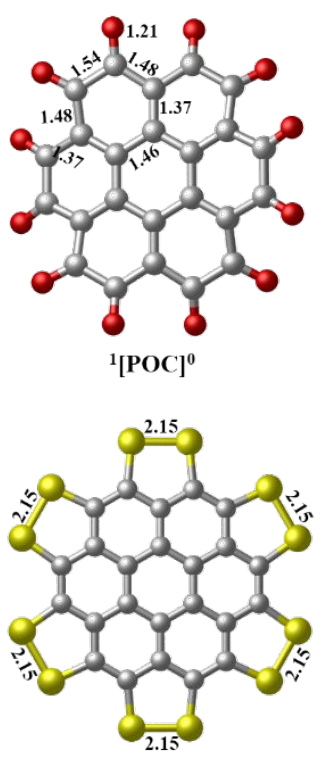

${ }^{1}$ PSC $^{0}$

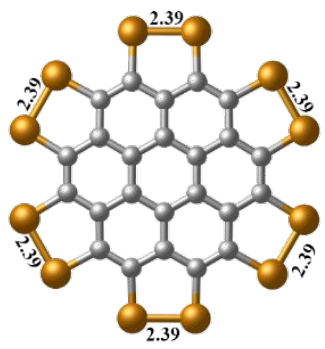

${ }^{1}[\mathrm{PSeC}]^{0}$

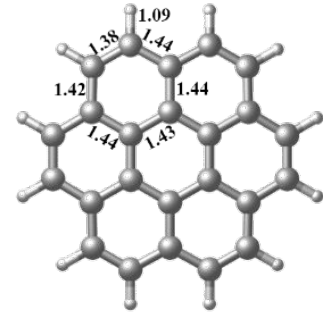

${ }^{2}[\text { Coronene }]^{1-}$
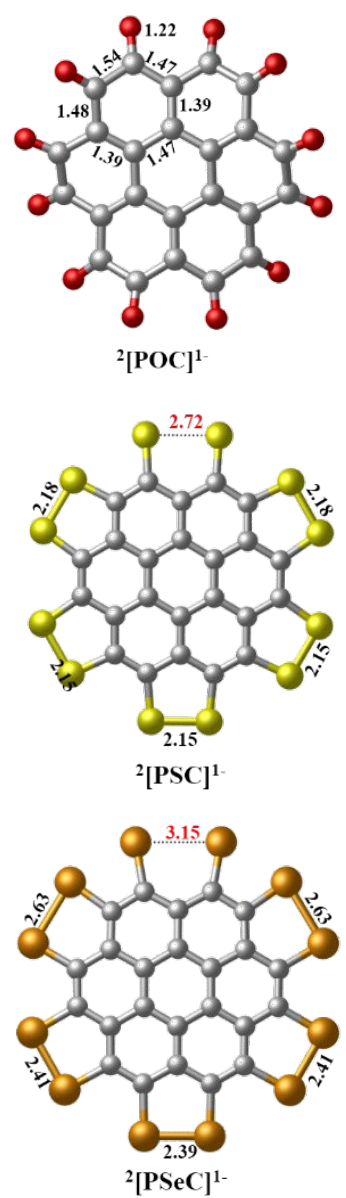

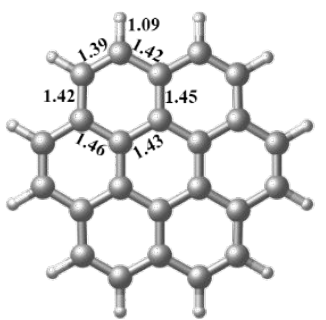

${ }^{3}[\text { Coronene }]^{2}$
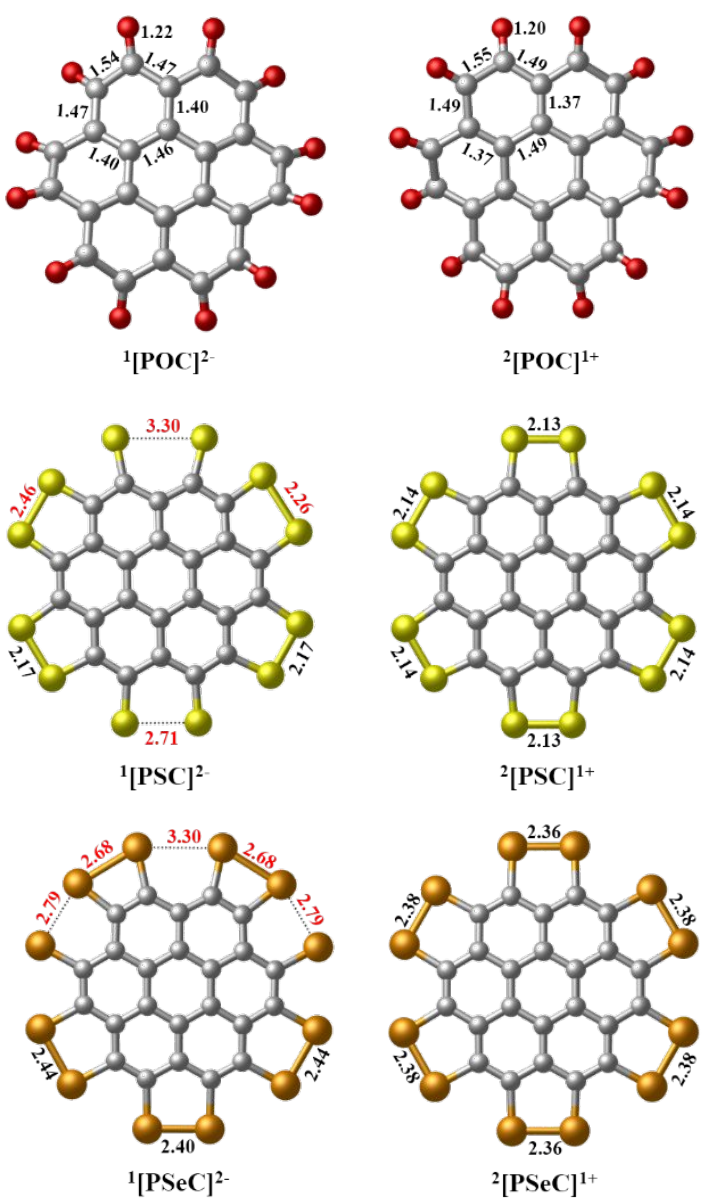

Figure S3. The optimized structures and selected bond lengths $(\AA)$ of all the studied singly and doubly charged systems at the B3LYP/6-31+G(d,p) level. 


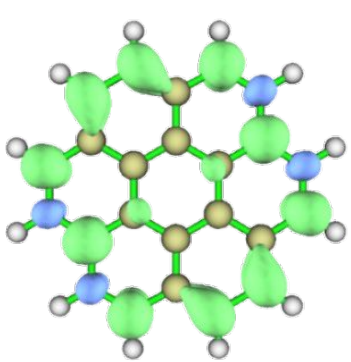

${ }^{2}[\text { Coronene }]^{1-}$

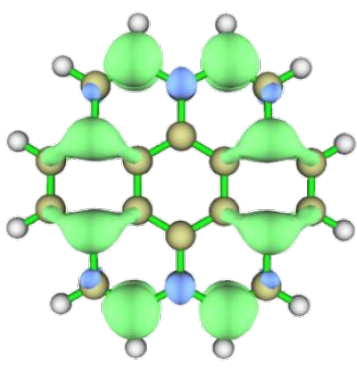

${ }^{2}[\text { Coronene }]^{1+}$
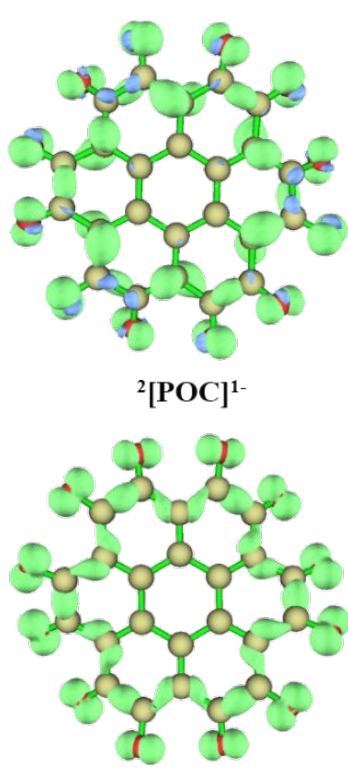

${ }^{2}[\mathrm{POC}]^{1+}$

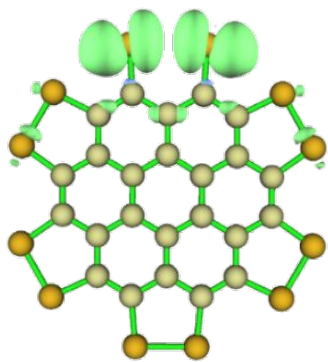

${ }^{2}[\mathrm{PSC}]^{1-}$

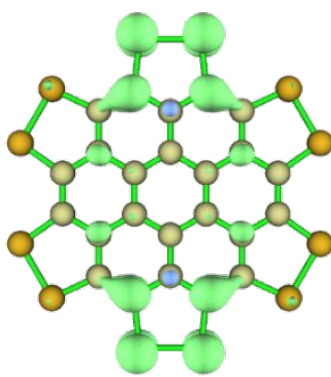

${ }^{2}[\mathrm{PSC}]^{1+}$

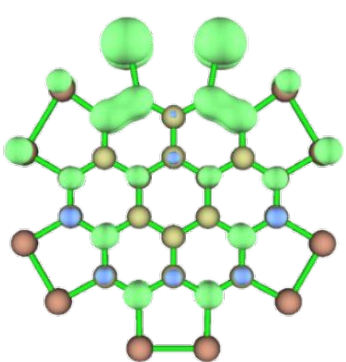

${ }^{2}[\mathrm{PSeC}]^{1-}$

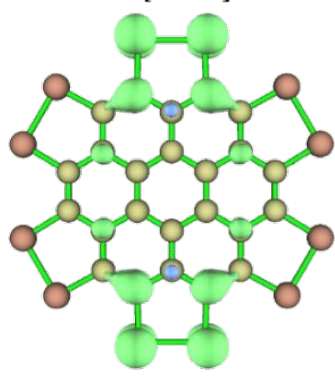

${ }^{2}[\mathrm{PSeC}]^{1+}$

Figure S4. The computed spin density distributions of the singly charged species of coronene, POC, PSC, and PSeC molecules. 


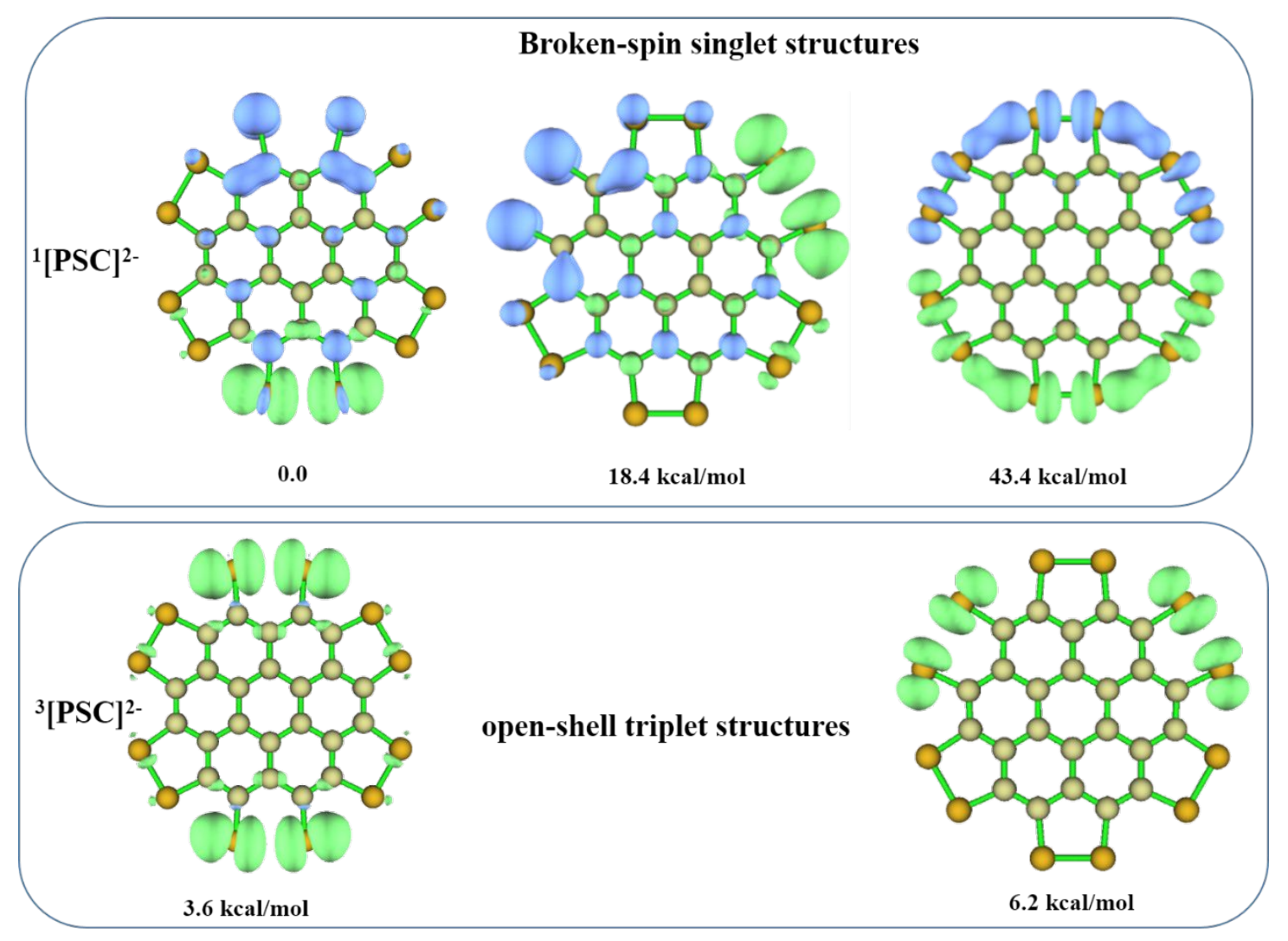

Figure S5. The spin density distributions of all the broken spin-symmetry (BS) $\left({ }^{1}[\mathrm{PSC}]^{2-}\right)$ and open-shell triplet structures $\left({ }^{3}[\mathrm{PSC}]^{2-}\right)$. 

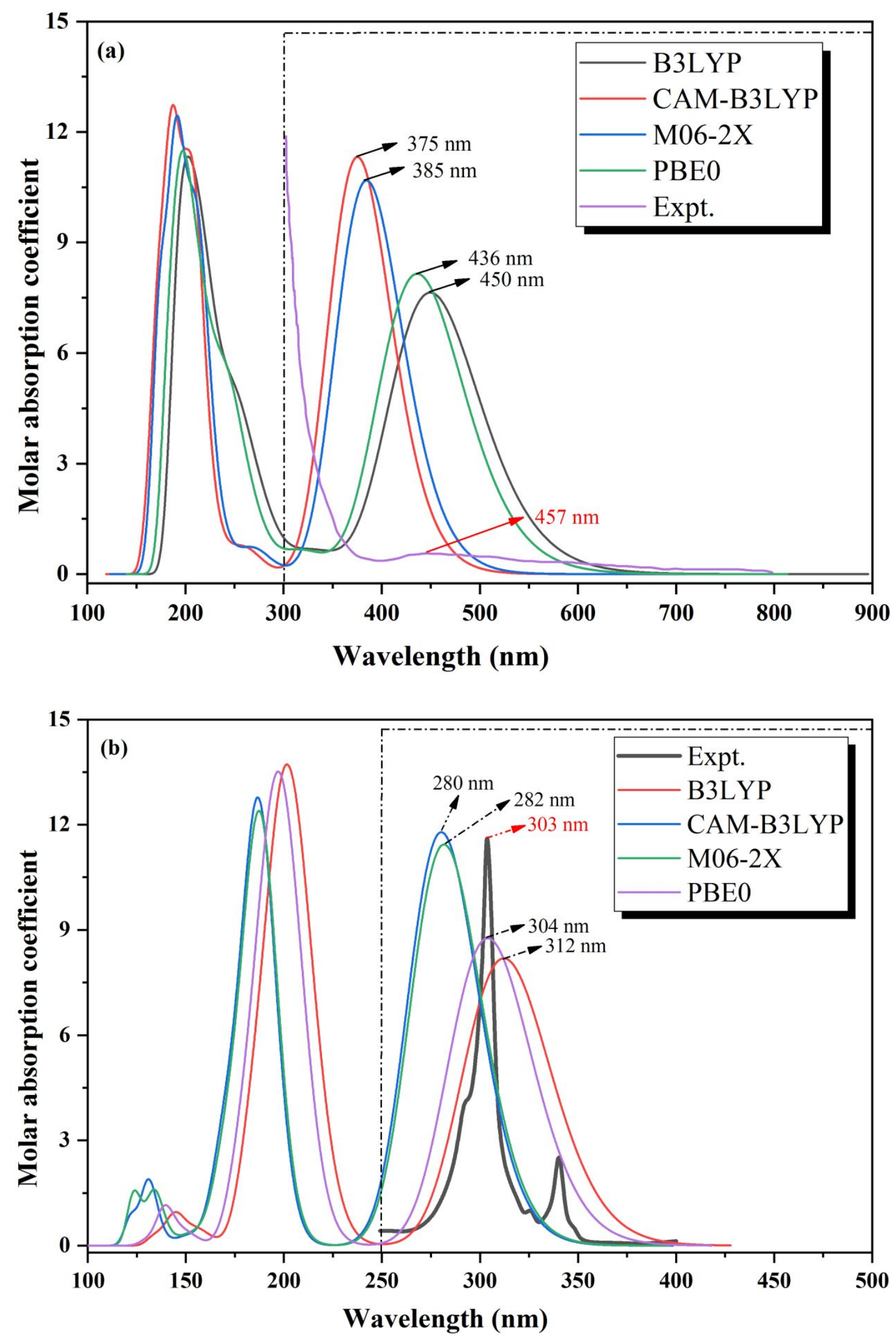


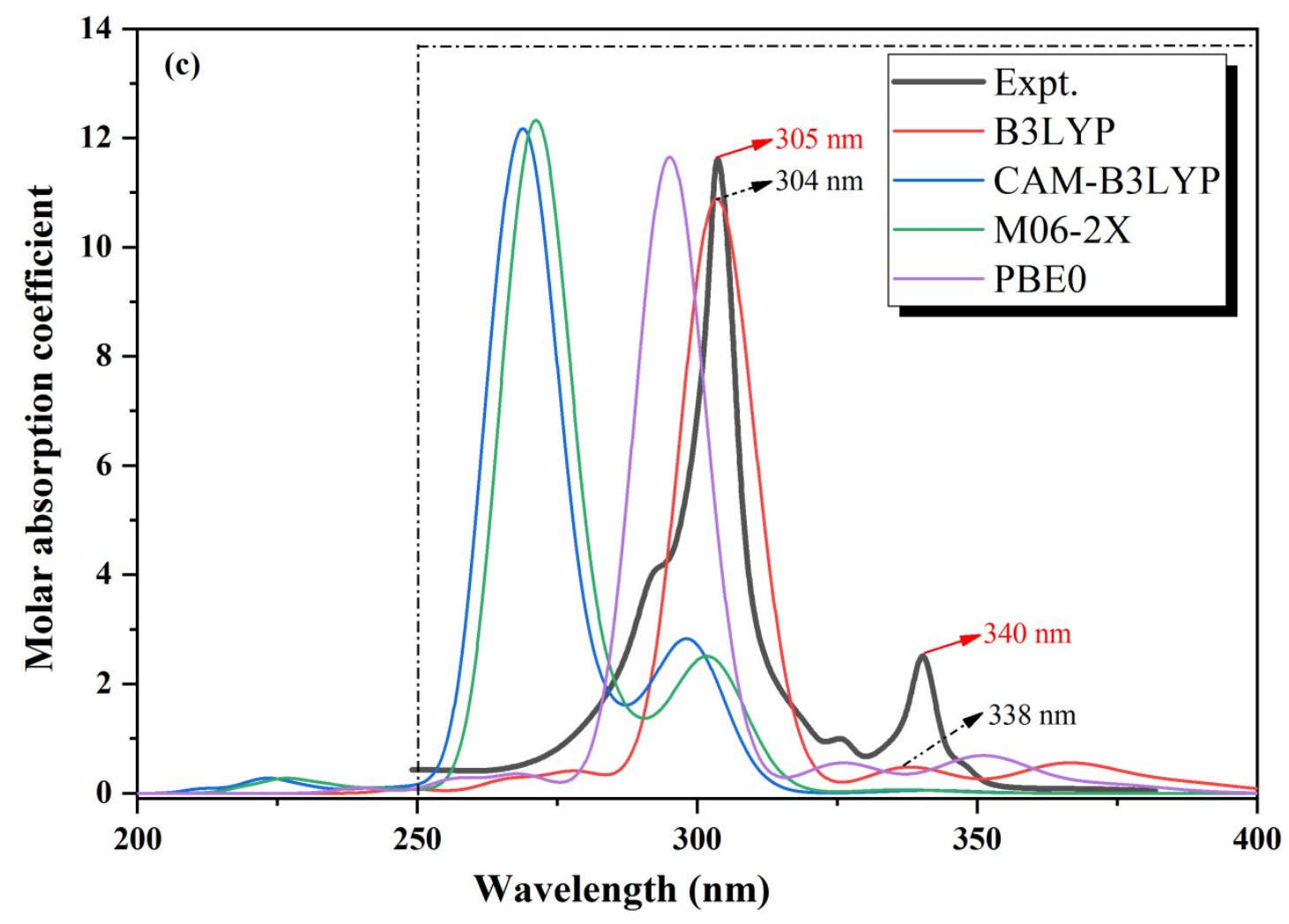

Figure S6 Simulated and experimental absorption spectra for the experimental reported PSC in N-methyl-2-pyrrolidone (NMP) media (a), coronene in cyclohexane (b) media ${ }^{62}$ and dimer absorption of coronene in cyclohexane media (c) by using TD-B3LYP, TDCAM-B3LYP, TD-M06-2X, and TD-PBE0 approaches, respectively, with the 6$31+\mathrm{G}(\mathrm{d}, \mathrm{p})$ basis set, on the basis of the ground-state geometry. The $\mathrm{x}$-axis range within the dotted line consists with the experiment. 

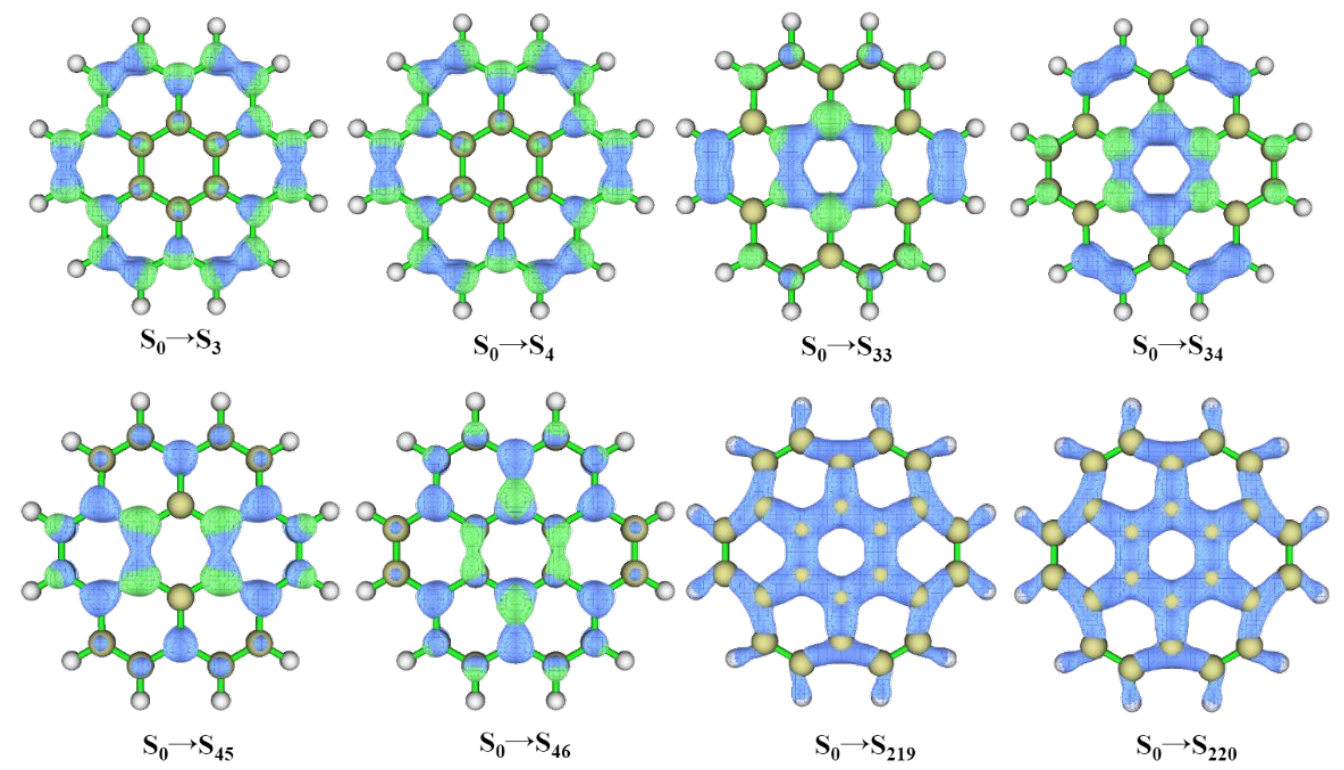

Figure S7. Predicted electron-hole distributions of coronene at the $\mathrm{S}_{0}$ state. Blue and green isosurfaces represent hole and electron distributions here and in subsequent Figures, respectively. 

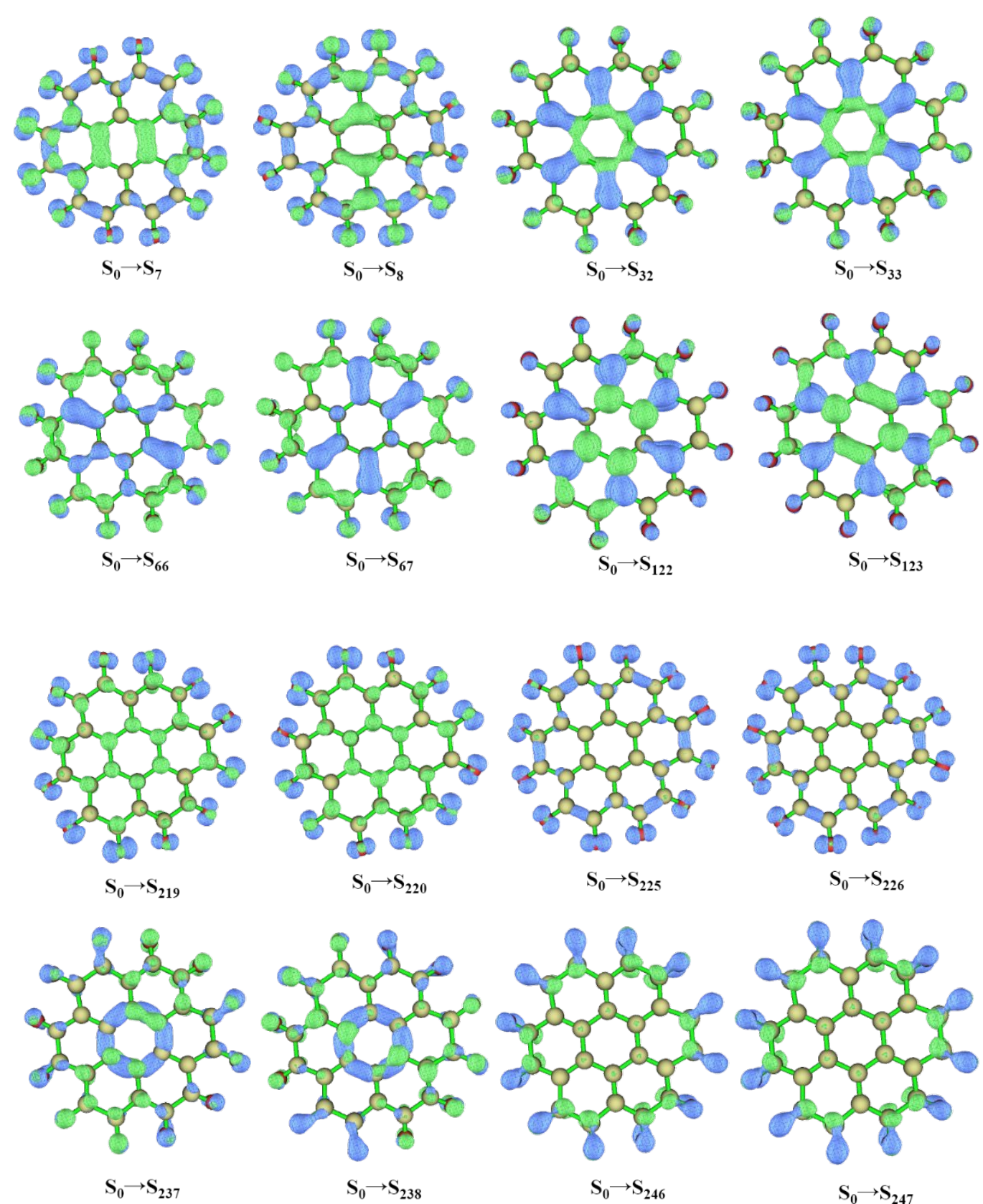

Figure S8. Predicted electron-hole distributions of POC in the $\mathrm{S}_{0}$ state. 

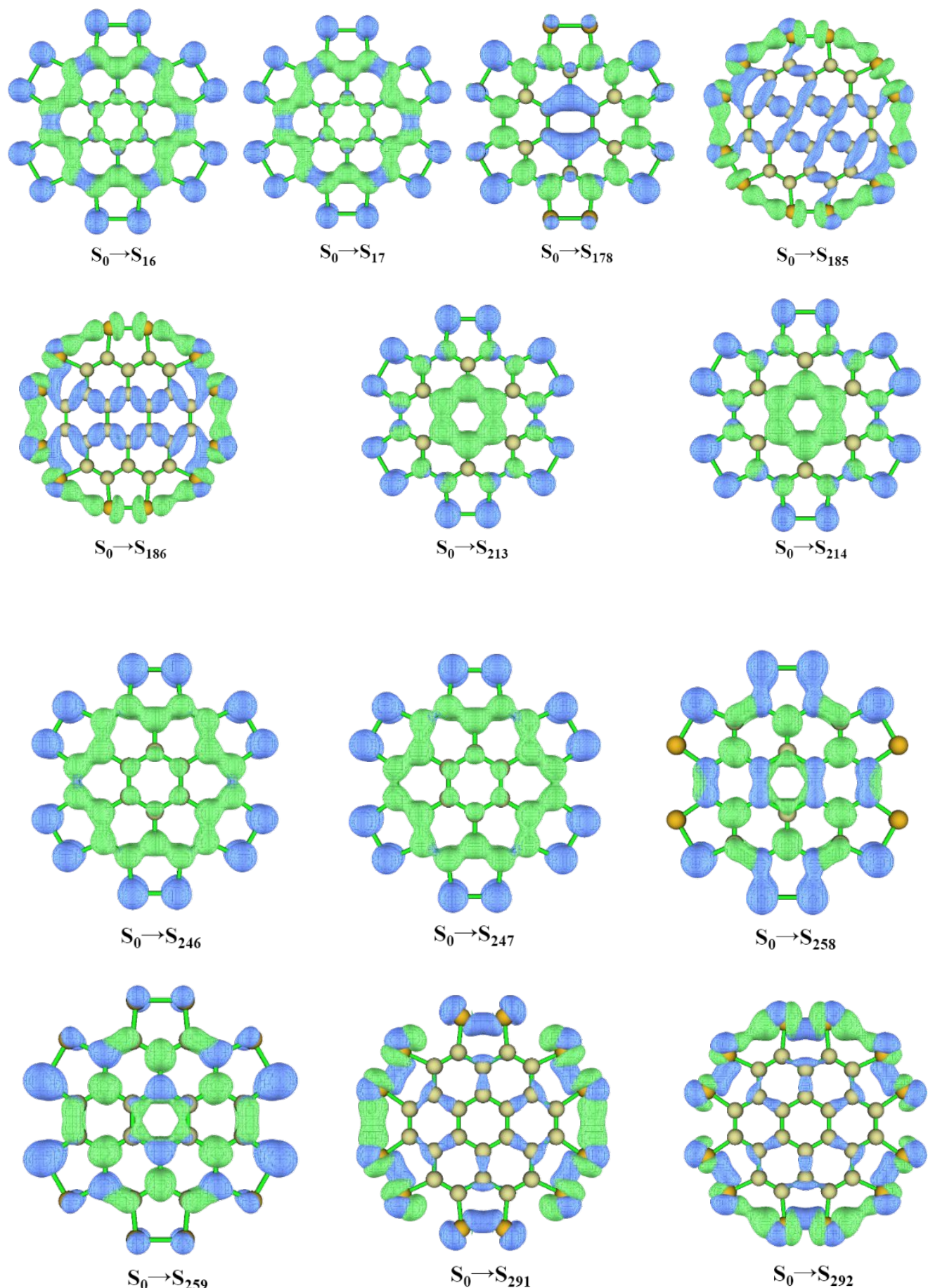

Figure S9. Predicted electron-hole distributions of PSC in the $\mathrm{S}_{0}$ state. 


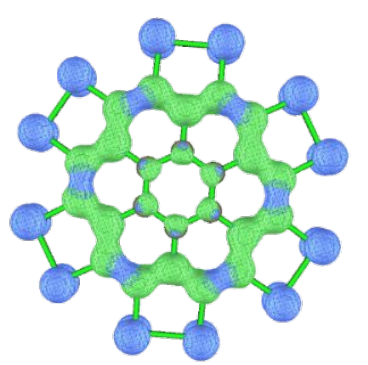

$\mathrm{S}_{0} \rightarrow \mathrm{S}_{17}$

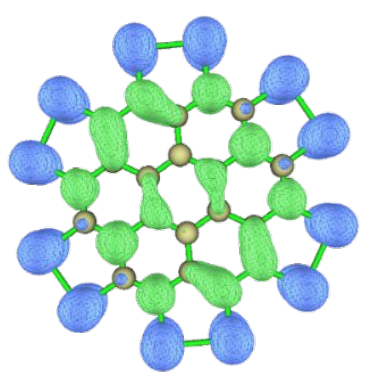

$\mathrm{S}_{0} \rightarrow \mathrm{S}_{66}$

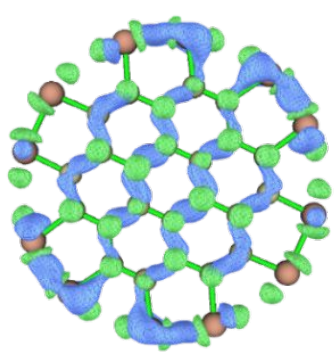

$\mathbf{S}_{0} \rightarrow \mathbf{S}_{245}$

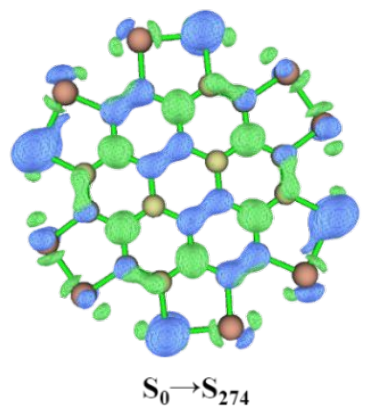

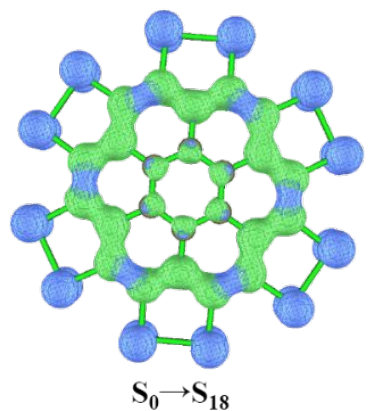

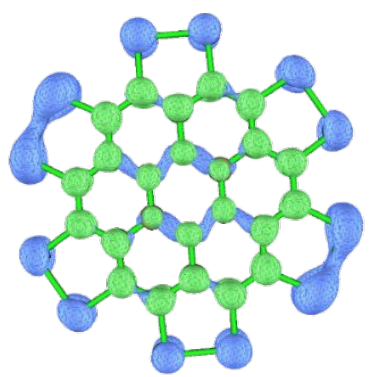

$\mathbf{S}_{0} \rightarrow \mathbf{S}_{229}$

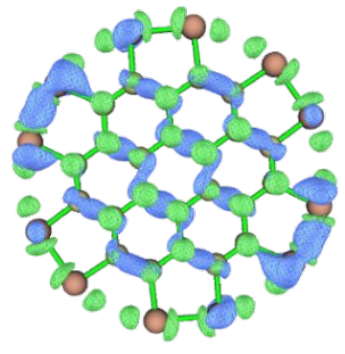

$\mathrm{S}_{0} \rightarrow \mathrm{S}_{246}$

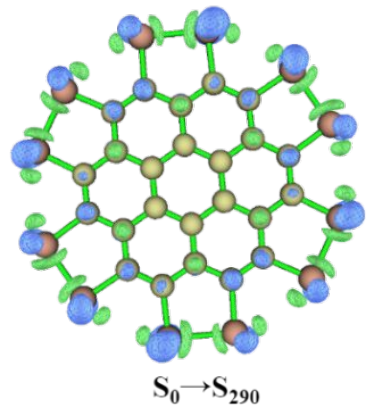

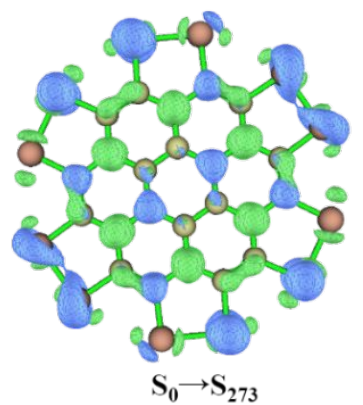
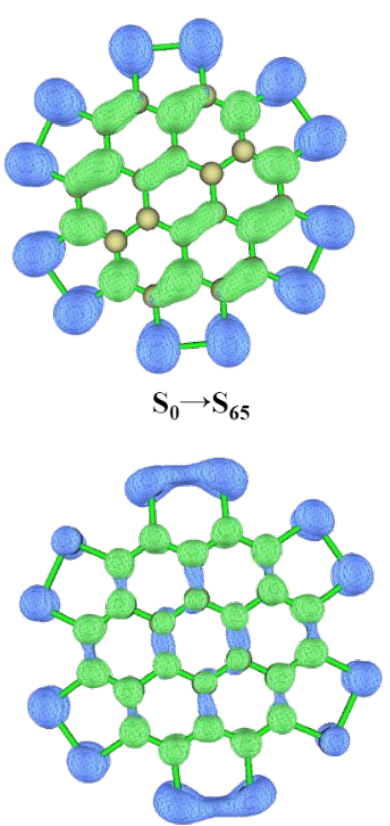

$\mathbf{S}_{0} \rightarrow \mathbf{S}_{230}$

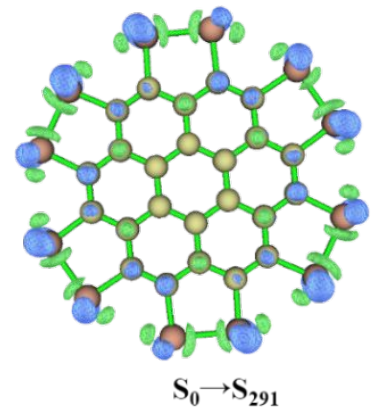

Figure S10. Predicted electron-hole distributions of $\mathrm{PSeC}$ in the $\mathrm{S}_{0}$ state. 
Table S1. Simulated and experimental energy levels of HOMO and LUMO, as well as the HOMO-LUMO (H-L) gap of the experimentally reported PSC by using B3LYP, CAM-B3LYP, M06-2X, and PBE0 approaches with the 6-31+G(d,p) basis set.

\begin{tabular}{lccccc}
\hline & B3LYP & CAM-B3LYP & M06-2X & PBE0 & Expt. $^{\mathrm{a}}$ \\
\hline HOMO & -5.28 & -6.43 & -6.32 & -5.42 & -4.23 \\
LUMO & -3.22 & -1.83 & -2.17 & -2.91 & -2.13 \\
H-L gap & 2.06 & 4.61 & 4.15 & 2.51 & 2.10 \\
\hline
\end{tabular}

${ }^{\mathrm{b}}$ The available experimental values of PSC. ${ }^{22}$ All energies are in $\mathrm{eV}$. 
Table S2. Predicted absolute adiabatic electron affinities (EA), ionization potentials (IP), and the available experimental values of coronene.

\begin{tabular}{cccccc}
\hline & $\mathrm{EA}_{1}$ & $\mathrm{IP}_{1}$ & $\mathrm{EA}^{\mathrm{a}}$ & $\mathrm{IP}^{\mathrm{a}}$ & $\mathrm{EA}_{\text {expt }}{ }^{\mathrm{b}}$ \\
\hline Coronene & 0.49 & 7.02 & 0.41 & 7.02 & $0.47 \pm 0.09$ \\
PSC & 2.75 & 6.32 & 2.53 & 6.40 & \\
PSeC & 2.98 & 6.14 & 3.01 & 6.21 & \\
\hline
\end{tabular}

a EA and IP are the theoretically predicted adiabatic electron affinities and ionization potentials from the recently published work. ${ }^{24}$

${ }^{b}$ The available experimental EA values of coronene. ${ }^{60}$ All energies are in $\mathrm{eV}$. 
Table S3. Wavelength $\left(\lambda_{\text {abs }}\right)$, state-state transition, and oscillator strength $(f)$ for the simulated absorption spectrum of coronene at the B3LYP/6-31G(d,p) level, together with the corresponding transition percent and the main electronic excitation character.

\begin{tabular}{cccccc}
\hline & $\lambda_{\text {abs }}[\mathbf{n m}]$ & Transition & $\boldsymbol{f}$ & Percent & Main character \\
\hline Coronene & 313 & $\mathrm{~S}_{0}-\mathrm{S}_{3}$ & 1.043 & 50.0 & $\pi \rightarrow \pi^{*}$ \\
& & $\mathrm{~S}_{0}-\mathrm{S}_{4}$ & 1.041 & 50.0 & $\pi \rightarrow \pi^{*}$ \\
& & $\mathrm{~S}_{0}-\mathrm{S}_{33}$ & 0.630 & 12.8 & $\pi \rightarrow \pi^{*}$ \\
& $\mathrm{~S}_{0}-\mathrm{S}_{34}$ & 0.629 & 12.8 & $\pi \rightarrow \pi^{*}$ \\
& $\mathrm{~S}_{0}-\mathrm{S}_{45}$ & 0.958 & 27.0 & $\pi \rightarrow \pi^{*}$ \\
& $\mathrm{~S}_{0}-\mathrm{S}_{46}$ & 0.954 & 26.9 & $\pi \rightarrow \pi^{*}$ \\
& & $\mathrm{~S}_{0}-\mathrm{S}_{219}$ & 0.094 & $39.43 \%$ & $\sigma \rightarrow \sigma^{*}$ \\
& & $\mathrm{~S}_{0}-\mathrm{S}_{220}$ & 0.094 & $39.4 \%$ & $\sigma \rightarrow \sigma^{*}$
\end{tabular}


Table S4. Wavelength $\left(\lambda_{\text {abs }}\right)$, state-state transition, and oscillator strengths $(f)$ for the simulated absorption spectrum of POC at the B3LYP/6-31+G(d,p) level, together with the corresponding transition percent and the main electronic excitation character.

\begin{tabular}{|c|c|c|c|c|c|}
\hline & $\lambda_{\text {abs }}[\mathrm{nm}]$ & Transition & $f$ & Percent(\%) & Main character \\
\hline \multirow[t]{16}{*}{ POC } & 787 & $\mathrm{~S}_{0}-\mathrm{S}_{7}$ & 0.106 & 49.8 & $\mathrm{n} \rightarrow \pi^{*}$ \\
\hline & & $\mathrm{S}_{0}-\mathrm{S}_{8}$ & 0.106 & 49.8 & $\mathrm{n} \rightarrow \pi^{*}$ \\
\hline & 408 & $\mathrm{~S}_{0}-\mathrm{S}_{32}$ & 0.379 & 48.2 & $\pi \rightarrow \pi^{*}$ \\
\hline & & $\mathrm{S}_{0}-\mathrm{S}_{33}$ & 0.379 & 48.3 & $\pi \rightarrow \pi^{*}$ \\
\hline & 299 & $\mathrm{~S}_{0}-\mathrm{S}_{66}$ & 0.291 & 33.3 & $\pi \rightarrow \pi^{*}$ \\
\hline & & $\mathrm{S}_{0}-\mathrm{S}_{67}$ & 0.289 & 33.1 & $\pi \rightarrow \pi^{*}$ \\
\hline & 219 & $\mathrm{~S}_{0}-\mathrm{S}_{122}$ & 0.716 & 45.2 & $\pi \rightarrow \pi^{*}$ \\
\hline & & $\mathrm{S}_{0}-\mathrm{S}_{123}$ & 0.716 & 45.2 & $\pi \rightarrow \pi^{*}$ \\
\hline & 173 & $\mathrm{~S}_{0}-\mathrm{S}_{219}$ & 0.137 & 11.2 & $\mathrm{n} \rightarrow \pi^{*}$ \\
\hline & & $\mathrm{S}_{0}-\mathrm{S}_{220}$ & 0.137 & 11.2 & $\mathrm{n} \rightarrow \pi^{*}$ \\
\hline & & $\mathrm{S}_{0}-\mathrm{S}_{225}$ & 0.137 & 11.5 & $\mathrm{n} \rightarrow \pi^{*}$ \\
\hline & & $\mathrm{S}_{0}-\mathrm{S}_{226}$ & 0.137 & 11.5 & $\mathrm{n} \rightarrow \pi^{*}$ \\
\hline & & $\mathrm{S}_{0}-\mathrm{S}_{237}$ & 0.095 & 7.9 & $\sigma \rightarrow \pi^{*}$ \\
\hline & & $\mathrm{S}_{0}-\mathrm{S}_{238}$ & 0.093 & 7.7 & $\sigma \rightarrow \pi^{*}$ \\
\hline & & $\mathrm{S}_{0}-\mathrm{S}_{246}$ & 0.073 & 5.2 & $\pi \rightarrow \pi^{*}$ \\
\hline & & $\mathrm{S}_{0}-\mathrm{S}_{247}$ & 0.073 & 5.2 & $\pi \rightarrow \pi^{*}$ \\
\hline
\end{tabular}


Table S5. Wavelength $\left(\lambda_{\text {abs }}\right)$, state-state transition, and oscillator strength $(f)$ for the simulated absorption spectrum of PSC at the B3LYP/6-31+g(d,p) level, together with the corresponding transition percent and the main transition character.

\begin{tabular}{|c|c|c|c|c|c|c|}
\hline & $\lambda_{\mathrm{abs}}[\mathrm{nm}]$ & Transition & $f$ & Percent(\%) & Main character & $\lambda_{\text {expt. }}[\mathrm{nm}]^{\mathrm{a}}$ \\
\hline \multirow[t]{8}{*}{ PSC } & 450 & $\mathrm{~S}_{0}-\mathrm{S}_{16}$ & 0.945 & 50.0 & $\pi \rightarrow \pi^{*}$ & 457 \\
\hline & & $\mathrm{S}_{0}-\mathrm{S}_{17}$ & 0.944 & 50.0 & $\pi \rightarrow \pi^{*}$ & \\
\hline & 203 & $\mathrm{~S}_{0}-\mathrm{S}_{178}$ & 0.201 & 3.3 & $\pi \rightarrow \pi^{*}$ & \\
\hline & & $\mathrm{S}_{0}-\mathrm{S}_{185}$ & 0.379 & 9.0 & $\sigma \rightarrow \sigma^{*}$ & \\
\hline & & $\mathrm{S}_{0}-\mathrm{S}_{186}$ & 0.380 & 9.1 & $\sigma \rightarrow \sigma^{*}$ & \\
\hline & & $\mathrm{S}_{0}-\mathrm{S}_{213}$ & 0.274 & 9.6 & $\pi \rightarrow \pi^{*}$ & \\
\hline & & $\mathrm{S}_{0}-\mathrm{S}_{214}$ & 0.273 & 9.5 & $\pi \rightarrow \pi^{*}$ & \\
\hline & & $\mathrm{S}_{0}-\mathrm{S}_{246}$ & 0.425 & 13.6 & $\pi \rightarrow \pi^{*}$ & \\
\hline
\end{tabular}

${ }^{\text {a }}$ The experimentally obtained absorption peak. ${ }^{21}$ 
Table S6. Wavelength $\left(\lambda_{\text {abs }}\right)$, state-state transition, and oscillator strength $(f)$ for the simulated absorption spectrum of $\mathrm{PSeC}$ at the B3LYP/6-31+g(d,p) level, together with the corresponding transition percent and the main electronic excitation character.

\begin{tabular}{|c|c|c|c|c|c|}
\hline & $\lambda_{\mathrm{abs}}[\mathrm{nm}]$ & State & $f$ & Percent(\%) & Main character \\
\hline \multirow[t]{12}{*}{$\mathrm{PSeC}$} & 463 & $\mathrm{~S}_{0}-\mathrm{S}_{17}$ & 0.9169 & 50.0 & $\pi \rightarrow \pi^{*}$ \\
\hline & & $\mathrm{S}_{0}-\mathrm{S}_{18}$ & 0.9165 & 50.0 & $\pi \rightarrow \pi^{*}$ \\
\hline & 321 & $\mathrm{~S}_{0}-\mathrm{S}_{65}$ & 0.2187 & 41.4 & $\pi \rightarrow \pi^{*}$ \\
\hline & & $\mathrm{S}_{0}-\mathrm{S}_{66}$ & 0.2186 & 41.4 & $\pi \rightarrow \pi^{*}$ \\
\hline & 213 & $\mathrm{~S}_{0}-\mathrm{S}_{229}$ & 0.2787 & 4.2 & $\pi+\sigma \rightarrow \pi^{*}$ \\
\hline & & $\mathrm{S}_{0}-\mathrm{S}_{230}$ & 0.2786 & 4.2 & $\pi+\sigma \rightarrow \pi^{*}$ \\
\hline & & $\mathrm{S}_{0}-\mathrm{S}_{245}$ & 0.7437 & 13.3 & $\pi+\sigma \rightarrow \pi^{*}+\sigma^{*}$ \\
\hline & & $\mathrm{S}_{0}-\mathrm{S}_{246}$ & 0.7424 & 13.3 & $\pi+\sigma \rightarrow \pi^{*}+\sigma^{*}$ \\
\hline & & $\mathrm{S}_{0}-\mathrm{S}_{273}$ & 0.9432 & 16.5 & $\pi \rightarrow \pi^{*}+\sigma^{*}$ \\
\hline & & $\mathrm{S}_{0}-\mathrm{S}_{274}$ & 0.9437 & 16.5 & $\pi \rightarrow \pi^{*}+\sigma^{*}$ \\
\hline & & $\mathrm{S}_{0}-\mathrm{S}_{290}$ & 0.3811 & 5.7 & $\pi \rightarrow \pi^{*}+\sigma^{*}$ \\
\hline & & $\mathrm{S}_{0}-\mathrm{S}_{291}$ & 0.3784 & 5.6 & $\pi \rightarrow \pi^{*}+\sigma^{*}$ \\
\hline
\end{tabular}

